\title{
Post Mortem Specimen of Ruptured Tubal Pregnancy: Symptoms Suggesting Irritant Poisoning.
}

\author{
By Wm. Alex. Mackay, M.D., Southport.
}

THE interest in this case lies principally in the history of the illness and of how the specimen came into my possession.

$\mathrm{Ou}$ the evening of March 4th last, I received a telephone request from the secretary of the Grocers' Association to attend, on their behalf, some miles from Southport, the post mortem examination of the body of a lady who had died suddenly shortly after eating some bloater paste, supplied by one of their members. The autopsy was to be made at the instance of the coroner, and in addition to the two medical gentlemen who had attended the case during life, I was to meet a distinguished bacteriologist.

The cause of death was supposed to be poisoning from the bloater paste. Just before the sectio the following history was obtained:-

Mrs. E. G., æt. 40, a healthy woman, made a meal of bloater paste, bread and butter, at 4-30 on March 2nd; at 7-30 she took some cold beef; at 10, part of an apple; and went to bed perfectly well. She wakened about 7-50 a.m.--her usual time-apparently well. At 8 a.m. she felt sick, and going to the bathroom, took a dose of Eno's fruit salt. Shortly after 8 she called over the banisters to the servant to prepare breakfast, mentioning the food she required-ham and eggs. She then complained of faintness, went giddy, and had nausea, followed by vomiting. Immediately thereafter she had diarrhøa. The diarrhœa continued with occasional vomiting. I cannot say what quantity of fæcal matter passed-the motions were liquid; apparently, however, her desire to have the bowels moved was so marked as to cause her anxiety that she could not relieve herself decently.

She was seen by her doctor from 10-30-11 a.m. She was then collapsed, pale and bloodless. The pupils were dilated. She was on her right side. The radial pulse was not perceptible, and only a faint impulse was felt in the carotid. Later a certain amount of cyanosis of the lips was noted, but the pallor persisted. She complained of some, but not severe, pain, and was troubled with thirst. In spite of stimulant treatment, including the administration of 20 ounces of normal saline subcutaneously, she died about 1 p.m.

The menstrual history could not be obtained before the body was opened. Later I learned from the husband that she was near a menstrual period, and that the one preceding had been less than usual; indeed he had made some chaffing remark to her of the possibility of pregnancy. 
The body was that of a very well-formed and well-nourished woman. Rigor mortis was well marked 45 or 46 hours after death. Pallor was marked.

On opening the abdominal cavity 2 or $3 \mathrm{lbs}$. of blood and clot were found lying free, and in the right oviduct was a ruptured gestation sac. In the blood-clot an embryo, measuring $18.5 \mathrm{~mm}$. was found. The uterus and tube were removed, and have been preserved. The uterus was nulliparous, and the sound passed in the cavity, after hardening, to 3 in. or thereby. The ruptured right tube was packed gently with cotton wool before being placed in spirit. In its present state it is some 2 in. in length.

The embryo was handed to Dr. Gowland, who is making certain researches. The measurement in the longest diameter is $18.5 \mathrm{~mm}$. and, as will be seen from the photograph handed round, flexion of the neck is still well marked. It is, I take it, at the end of the 6th or the beginning of the 7th week.

Cases of sudden death after rupture of an ectopic pregnancy have unfortunately occurred from time to time. Bland Sutton, in his book on diseases of the Fallopian tube, mentions the case of an English actress, who died suddenly while taking an ice in a café in the Bois de Boulogne. Poisoning was suspected. The death later was proved to be due to a ruptured ectopic pregnancy. $\mathrm{He}$ states that many cases of ruptured ectopic pregnancy have only been revealed by dissection instituted by the coroner's order.

The isthmus is supposed to be the most dangerous site for a tubal pregnancy. Our present case is purely tubal-in the centre of the tube at that.

With regard to the possibility of operation had the case been recognised, one must remember that the patient lived some miles from surgical aid, and that $2 \frac{1}{2}$ hours passed between the doctor's visit and death-a short period. Had the gravity been recognised at the time of rupture a period of four to five hours should have sufficed to save life. Technical difficulties the operation itself did not exist. 